\title{
Statistics Analysis of Decimetric Type III Bursts, Coronal Mass Ejections and $\mathbf{H} \alpha$ Flares
}

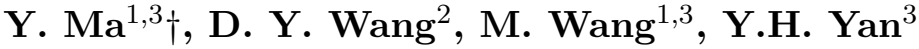 \\ ${ }^{1}$ Yunnan Observatory, Chinese Academy of Sciences, P.O. Box 110, 650011 Kunming, \\ P.R. China \\ email: mayuanf@public.km.yn.cn \\ ${ }^{2}$ Purple Mountain Observatories, Chinese Academy of Sciences, 210008 Nanjing, P.R. China \\ ${ }^{3}$ National Astronomical Observatories, Chinese Academy of Sciences, 100012 Beijing China
}

\begin{abstract}
The Statistics analysis of decimetric type III bursts, coronal mass ejections (CMEs) and $\mathrm{H} \alpha$ flares are carried out. The relevant radio events observed from the $625-1500 \mathrm{MHz}$ spectrograph at the Yunnan Observatory during the 23rd solar cycle. It is found that the relation between the decimetric type III bursts and CMEs is not closer than that between the type II radio bursts and CMEs; All $\mathrm{H} \alpha$ flares generated decimetric type III bursts and correlated with CMEs are all gradual flares. The higher the energy of the flare correspond to the faster the initial velocity of the CMEs.
\end{abstract}

Keywords. Sun: activity, CMEs, radio radiation

\section{Introduction}

Is there any relation between the small-scale decimetric type III bursts and the largescale CMEs? If any, how close is it? for this, the authors studied the relation between the type III bursts and CMEs and $\mathrm{H} \alpha$ flares, acquiring some physical information and approaching the cause of CMEs.

\section{Data processing}

264 decimetric type III bursts were recorded during the 23rd solar cycle from 625$1500 \mathrm{MHz}$ spectrograph at the Yunnan Observatory. If the comparison is made only from the angle of time, there are 138 type III bursts correlating to the CMEs, amounting to $52 \%$ of total bursts, and 90 type III bursts corresponding to the $\mathrm{H} \alpha$ flares. However, since the radio spectrograph has no spatial resolution one can not determine the active region. Therefore, if the relation between the type III bursts and CMEs is considered only from the angle of time, the statistical error would be very large because these type III bursts and CMEs do not come from the same source position though they occur simultaneously. For this, the authors only selected the type III bursts which occurred with the CMEs and $\mathrm{H} \alpha$ flares simultaneously for statistics. The identification and selection of the data are carried out according to the following steps: We choose the statistical data in the following procedure. An event of decimetric type III burst is taken firstly, and then to find CME data within the time interval (40 minutes) from LASCO. However, only the

$\dagger$ Present address:Yunnan Observatory, Chinese Academy of Sciences, P.O.Box 110, 650011 Kunming, P.R. China. 
position of CME data is similar to the source of $\mathrm{H} \alpha$ flares, generated the type III burst, to be adopted.

\section{Analysis Results and Discussions}

1) The statistics of the events which occurred within 2 hours before the CMEs are collected, with 5 minutes being the time interval. The correlation between the decimetric type III bursts and the CMEs is $52 \%$ when the source position is not determined and is $28 \%$ after the source position is determined; most of the type III bursts occur in 45 minutes before the CMEs. 2) The $\mathrm{H} \alpha$ flares which generated decimetric type III and correlated to the CMEs are all the gradual flares. $82 \% \mathrm{H} \alpha$ flares erupt before CMEs, with the occurrence being in first 33 minutes on average. Only $18 \%$ of $\mathrm{H} \alpha$ flares occur after CMEs, with the occurrence being in the last 29 minutes on average. 3) The initial frequencies of $64 \%$ of decimetric type III bursts occur at $700 \mathrm{MHz}$; most of the frequency drift rates of the type III bursts corresponding to the CMEs occur at $100 \mathrm{MHz} / \mathrm{s}$ and the next ones in number occur at $700 \mathrm{MHz} / \mathrm{s}$. 4) The faster the initial velocity of the CMEs, corresponds to the higher the energy of the $\mathrm{H} \alpha$, but the acceleration or de-acceleration of CME does not depend to the energy of $\mathrm{H} \alpha$ flare. 5) 25\% of the type II bursts correspond to the type III bursts and $50 \%$ of the soft X-ray events correspond to the type III bursts.

It can be obtained from the above statistics analysis that the gradual flares correlating to the CMEs occur before and after the CMEs, however, most of CME occur later than $\mathrm{H} \alpha$ flares. As for the flares after the CMEs, the reason may be that the distance from the magnetic reconnection place to the photosphere, along which the electron beam propagated inwards is longer than that from the magnetic reconnection place to the corona, therefore the $\mathrm{H} \alpha$ flares occur later than the CMEs.

The authors hold that the high-energy electrons of the decimetric type III bursts should be accelerated in the process of the flares and they escape outwards along the open magnetic lines of force in the corona, thereby producing the type III bursts with extremely fast frequency drift while the type II radio bursts are accelerated by the shock waves produced when the CMEs propagate outwards. Thus, the type II bursts have the slow frequency drift of the propagating velocity of the shock waves (Nelson and Melrose, 1985). According to the above-mentioned points of view the correlation between the CMEs and the type II radio bursts should be far much greater than that between the CMEs and the type III radio bursts. The results obtained by the authors are that the correlation between the CMEs and the type III radio bursts is less than $40 \%$, much less than the correlation, $60 \%$ or $80 \%$. Between the CMEs and the type II radio bursts, which is in accordance with the above-mentioned model.

\section{Acknowledgements}

This research is supported by the Ministry of Science and Technology of China grant No. G2000078403, and also supported by the national NSF of China (grant Nos. 10473020 and 10333030).

\section{References}

Gopalswamy, Y. S. and Kaiser, M. L. 2001, ApJ. 548, 94

Jackson, B. V., Sheridan, K. V., Dulk, G. A. et al. 1978, Solar Phys. 3, 241

Michalek, G., Gopalswamy, N., Reiner, M. et al. 2001, American Geophysical Union, Spring Meeting., 5

Nelson, G. J. and Melrose, D. B 1985, Solar radiophysics., 333 